\title{
OPEN ACCESS BARRIERS: AN ACTION RESEARCH
}

\author{
Mathias Klang \\ University of Lund, Sweden
}

\begin{abstract}
ICT has provided the infrastructure to enable easy access to scientific information. Despite this, libraries are suffering from the rising of journal subscriptions. Additionally, the structure of scholarly publications is creating a wasteful situation where publicly funded research is being paid for several times over. University libraries are struggling to deal with these new realities at the same time as they provide a level of service with acceptable access to publications. The work of librarians is being heavily affected by the influence of copyright and licensing which together are creating barriers to open access. The work in this chapter draws from an action research in progress undertaken by Lund's University in order to explore the barriers to open access to scientific research output in Sweden.
\end{abstract}

Keywords: Open access, action research, scholarly publication

\section{Introduction}

In the early stages of the development and dissemination of ICT as a communications infrastructure a great deal of optimism about its potential to provide an equal open democratic access to the public sphere was expressed. The technology in focus in this chapter is Internet based communications technology. Much of the discussion in the early stages of the development of the Internet dealt with its uncontrollable nature. For those outside traditional power structures it was hailed as an important alternative to achieve wide spread communication while many of those inside the power structures were concerned with the negative effects of this new found freedom [14].

Both the cyberlibertarians and the cyberpaternalists agreed that the Internet provided a global information and communications infrastructure and that it could be used to create a public sphere [14]. In addition to this, a fundamental basis for such a development was the access to information and, in particular, access to scientific information.

Yet while the developments in communications technology provides an infrastructure it is not without limitations. Developments in technology make access to information possible and yet there still remain great barriers to access to scientific information. These barriers have moved from the technical to the legal 
and administrative and in the field of free access to scientific journals this is expressed in the form of copyright law and licenses.

The use of this barrier to open access (OA) has created a wasteful situation where important scientific data is locked away and were universal access to vital information remains unfulfilled. The purpose of this chapter is to explore the effects of copyright law and licenses on the ability to provide open access to scientific information. This chapter will use semi-structured interviews which are used to illustrate the way in which different actors are attempting to adapt to the new realities of the technical, legal, and academic structure in order to achieve a greater efficiency in the access to scientific knowledge.

The following section will describe the fundamentals of open access and scientific publications. This will be followed by an explanation of the methodology used in this chapter and the results of the method. This will be followed by an analysis of the results and the chapter will close by drawing some conclusions on the results.

\section{Theory}

The Internet was developed as an open (end-to-end) system with a focus on the non-discriminatory transportation of content. In other words the Internet is not concerned about the content but focuses on delivering from sender to receiver. Compared with print, such a system radically alters the ability of users to access information in addition to this the creation of digital copies is associated with low costs. Lastly, providing a digital copy online ensures that multiple users can access the same information without prejudicing each other's ability to do so, and without incurring additional costs for the sender. In this way, Internet technology has radically disrupted $[11,14]$ the non-digital models for the publishing scientific journals. This is recognized in the 2003 Berlin Declaration, which states in its preface:

"The Internet has fundamentally changed the practical and economic realities of distributing scientific knowledge and cultural heritage. For the first time ever, the Internet now offers the chance to constitute a global and interactive representation of human knowledge, including cultural heritage and the guarantee of worldwide access."

The barriers to knowledge therefore shift from being a barrier of being able to physically access and interpret the scientific knowledge to being one of legal access to the knowledge. This does not mean that other barriers have completely disappeared. Ability to use the new technology and to interpret scientific data is by no means universal. However the main focus has shifted from transportation to law. The basis of this new barrier to access lies in the use of copyright law and licensing.

The technology of the printed word has long held a unique position in the dissemination of scientific knowledge; its most specialized form, the scientific journal, was established 300 years ago [23]. Since the establishment of the 
scientific journal as a publishing model the popularity and growth has been steady. Even so, in the last half century the growth in the number of journals has been unprecedented [23]. According to Tenopir [20] there were almost 50,000 academic journals in 2003.

In addition to the growth in the number of journals there has also been a concentration of media, which has entailed a decrease in the number of independent publishers. In other words, fewer publishers are producing a larger number of journals. The situation is made more precarious by the evolution of the so-called serials crisis [21]; this is the rapidly rising cost of academic journals. It is generally recognized that the cost of scientific journals has risen at a rate that exceeds that which can be motivated by inflation [5, 6, 13, 17]. As a result university libraries are struggling to provide researchers with access to the necessary journals.

The recent development of the open access ideology comes from two primary driving forces: the desire for openness and the need to resolve the so-called serials crisis.

This has led to multiple responses in order to achieve greater levels of open access $[11,23]$ :

(1) Open access Journals;

(2) Institutional repositories;

(3) Policy statements (declarations); and

(4) Changes in research funding policy.

The launch of open access journals has come from a few different directions. Some have been developed as new journals, some are established journals that have changed their policy to become open access, and finally there are the declarations of independence where journal editors or whole editorial boards have resigned in protest and launched open access alternatives.

An important aspect to remember about the open access journal is that there are several alternative forms. For example the journal content is made freely available to readers. In the so-called green route the material is made freely available to readers because the journal itself has an alternative method of financing its existence (for example advertising, grants, unpaid labour). While in the "gold" route the author pays the publisher a fee prior to publication and the material is then free to all readers.

A practice closely connected to the open access journal is self-archiving. This system has been developed to enable the archiving of content on an institutional level. Self-archiving can entail practices such as the author maintaining his/her own publications via a personal web page and the use of institutional repositories. From the point of view of an organisation, longevity and searchability of the institutional repositories, for example in libraries, have been recognized as far superior.

From these developments a level of institutional policy support has materialized in the form of different declarations in favour of open access systems. 
Some of the more important such declarations are the Declaration on Science and the Use of Scientific Knowledge (UNESCO-ICSU World Conference on Science, 1999), a United Nations Economic and Social Council ministerial declaration (2000), the Declaration of Havana (2001), the Budapest Open Access Initiative (2001), the Third World Academy of Sciences (TWAS) issued the Beijing Declaration on scientific advancement, openness, and cooperation (2003), the Berlin Declaration on Open Access to Knowledge in the Sciences and Humanities (2003), the UN World Summit on the Information Society approved a Declaration of Principles and Plan of Action (2003), the Valparaiso Declaration for Improved Scientific Communication in the Electronic Medium (2004), and Ministerial representatives from 34 nations to the Organisation for Economic Co-operation and Development (OECD) issued the Declaration on Access to Research Data From Public Funding (2004).

While these declarations vary in exact content they all intend to provide greater levels of access to scientific publications. If we take the wording of the Berlin Declaration as an example we see that open access contributions must satisfy two conditions:

1. The author(s) and right holder(s) of such contributions grant(s) to all users a free, irrevocable, worldwide, right of access to, and a license to copy, use, distribute, transmit, and display the work publicly and to make and distribute derivative works, in any digital medium for any responsible purpose, subject to proper attribution of authorship (community standards, will continue to provide the mechanism for enforcement of proper attribution and responsible use of the published work, as they do now), as well as the right to make small numbers of printed copies for their personal use.

2. A complete version of the work and all supplemental materials, including a copy of the permission as stated above, in an appropriate standard electronic format is deposited (and thus published) in at least one online repository using suitable technical standards (such as the open archive definitions) that is supported and maintained by an academic institution, scholarly society, government agency, or other well-established organization that seeks to enable open access, unrestricted distribution, inter operability, and long-term archiving.

Declarations such as these have been echoed in many other professional organisations and university bodies. Additionally they have collected signatories and supporters around the globe. The effect has been to prove that there is a wide support for the principles of open access.

These practical and political developments are also being reflected in the ways in which scientific research is being funded. The best example of such action is the policy changes carried out in 2005 by the Wellcome Trust when it began to implement its new open-access mandate for Wellcome-funded research. 
As this all too brief overview of open access developments shows, many of the actors involved in the production and dissemination of scientific knowledge are working towards creating an environment where open access becomes a norm.

\section{Librarians \& action research}

As we have seen above there is a need to ensure that the access to scientific publications is enhanced through open access since the alternative is proving to be too costly and too wasteful a system. In attempting to operationalise the goals and visions expounded in the different declarations it has fallen upon the university libraries to ensure that open access visions are made practicable. Without the ability of these information systems specialists to provide solutions the individual authors can only make piecemeal attempts in securing access to scientific information.

The initial steps that have been taken have focused upon creating institutional repositories [1, 3, 10] and ensuring that the material being made available by individual publishers and journals was being made available to a wider audience. The next step in the process was to convince authors of the viability, sustainability and value of the open access alternatives. Much of the latter work has been to show the resulting impact of publishing in open access environments [7, 9].

A recent project launched at the University of Lund in Sweden has taken the action research approach in order to actively explore and impact on the use of open access. Action research builds upon a close collaboration between the researcher and the reality that is being studied. There are several different forms of action research recognised within IS research [4]. However, as Holwell [12] points out, these forms all share certain characteristics to enable them to fall into the action research field. These common characteristics are (1) that the researcher is immersed within the research object, (2) the work is not driven by the needs of the researcher, (3) problems arise from the local context, (4) descriptions and theories are built and tested empirically and iteratively within the local context $[2,4,16$, 22].

The process of action research consists of re-iterating a research cycle consisting of (1) identifying the problem (diagnosis), (2) creating goals/planning, (3) implementation, and (4) evaluation [19]. The method is not uncontroversial. Baskerville and Wood-Harper [4] identify three important pitfalls (issues of impartiality, discipline, and context-dependency), which the researcher using this method must be aware of, and address. Action research was chosen as the method for this research since the method supports both positivist and interpretavist approaches [15] irrespective of this the results arising from this method can be used "across varying epistemologies, ontologies, and methodologies" [8]. In addition to this, the method is openly an approach that attempts to develop important contributions to practice while carrying out research within that practice, and at the same time providing valuable insights to the research community [18]. 
In the aforementioned project, the university library of Lunds University began by conducting a survey among all the university libraries in Sweden in order to find out more about their procedures in dealing with copyright law and licensing in relation to achieving a greater level of open access. The initial survey phase will then be followed up by the creation of best practice models for the university libraries. These models are intended to create a consensus among the university libraries and also to provide a better basis of understanding of the varying problems when dealing with copyright and licensing. In addition to this it is hoped that the development of consensus among university libraries will provide researchers and authors of scientific articles with a clearer image of the way in which open access solutions can enable them to reach a wider audience for their work.

The purpose of the project is to develop competencies and provide legal support in relation to copyright issues brought about by open access publishing. The primary focus group is researchers, administrators, research funding offices, and universities in general.

The two-year project began in September 2007 with preliminary planning and research for the project and the data collection phase of the project. The resulting plan was a long-term research project with several concrete short-term goals. The project also has a goal to provide legal support throughout the project.

In the aforementioned project the university library of Lunds University has begun by conducting a survey among all the university libraries in Sweden in order to find out more about their procedures in dealing with copyright law and licensing in relation to achieving a greater level of open access. The initial survey phase will then be followed up by the creation of best practice models for the university libraries. These models are intended to create a consensus among the university libraries and also to provide a better basis of understanding of the varying problems when dealing with copyright and licensing. In addition to this it is hoped that the development of consensus among university libraries will provide researchers and authors of scientific articles with a clearer image of the way in which open access solutions can enable them to reach a wider audience for their work.

Action research is a reflective process of progressive problem solving led by individuals working with others in teams or as part of a "community of practice" to improve the way they address issues and solve problems. Action research can also be undertaken by larger organisations or institutions, assisted or guided by professional researchers, with the aim of improving their strategies, practices, and knowledge of the environments within which they practice.

The action component of this research stems from the need not only to conduct empirical research and report findings but also to actively present changes locally and lobby for policy changes nationwide. The process will be carried out iteratively by conducting interviews with different groups affected by the open access problem complex and successively attempting to overcome each groups 
problems by suggesting best practice models and then returning to evaluate the results of these changes within the organisation.

\subsection{The interviewees}

University College of Borås: The University College of Borås, UCB, consists of six academic Schools. In total, UCB has now 11,000 registered students, where approximately 6.300 are (registered) Degree programme students. The university has 536 employees. ${ }^{1}$ The interview at the University College of Borås was conducted on the 20 February and lasted a little over an hour.

Linköping University: The university is organized in four faculties and 14 departments. It has a student population of 25,000 and 3,500 employees. ${ }^{2}$ The interview at the university was conducted on the 21 February and lasted an hour and a half.

Jönköping University: Jönköping University Foundation is one of three independent institutions of higher education (not state-owned) in Sweden that are entitled to offer postgraduate programmes. Research and education is carried out in four schools: Jönköping International Business School, School of Education and Communication, School of Engineering and School of Health Sciences. ${ }^{3}$ The interview was carried out on the 22 February and lasted one hour.

Chalmers Technical University: Number of students 6,343 and number of teachers and researchers $1,433 .{ }^{4}$ The interview was conducted on the 26 February and lasted 75 minutes.

Stockholm University: Stockholm University has over 50,000 undergraduate and master's students, 1,800 doctoral students and 5,200 employees. ${ }^{5}$ The interview was conducted on the 27 February and lasted 50 minutes.

The University of Gävle is comprised of six departments that offer approximately 50 degree programmes and 800 elective courses. There are approximately 13,000 registered students and approximately 800 employees. ${ }^{6}$ The interview was conducted on 8 April and lasted almost one hour.

The University of Halmstad has around 50 programmes, 10,000 students and around 550 employees. ${ }^{7}$ The interview was carried out on 10 April and lasted 50 minutes.

The Göteborgs University has about 50,000 students and more than 5,000 employees. ${ }^{8}$ The interview was conducted on 11 April and lasted 75 minutes.

\footnotetext{
${ }^{1}$ http://www.hb.se/english/ucb/

${ }^{2}$ http://www.liu.se/en/presentation/

${ }^{3}$ http://www.hj.se/doc/4417

${ }^{4}$ http://www.chalmers.se/en/sections/about_chalmers/facts_and_figures_1

${ }^{5} \mathrm{http}: / /$ www.su.se/pub/jsp/polopoly.jsp?d=3807\&a=26335

${ }^{6}$ http://www.hig.se//ufk/is/introduction.html

${ }^{7}$ http://www.hh.se/omhogskolan.10.html

${ }^{8}$ http://www.gu.se/omuniversitetet/
} 
Umeå University has 29,000 students and close to 4,000 employees. The university offers 1,900 courses and 240 programmes. The interview was conducted on 12 April and lasted one hour.

\section{4. $\quad$ Results}

The resulting nine hours of interviews reflect different areas of concerns presented by the interviewees. The main thrust of these concerns can be divided into the following groups:

1. Copyright Concerns;

2. Research Culture;

3. Administrative Concerns;

4. University Context;

5. Legal Culture; and

6. Information Needs.

In order to preserve the anonymity of the interviewees the material presented in the analysis will not be identifiable with any particular university.

\section{Analysis}

All the universities involved identified copyright as a major source of concern in their everyday work. The main source of trouble was the lack of information about the way in which copyright and copyright licenses could and should be interpreted in the workday experience of the library.

The concern with copyright was more serious among the libraries that had a larger amount of researchers and a well functioning self-archiving system. However, even the smaller universities expressed concern with the complexities of copyright in relation to their everyday work.

The lack of information was made more acute since the libraries experienced that they are receiving more copyright and licensing questions from researchers that they were expected to answer with a fair level of certainty.

Some of the universities also expressed concerns that they did not have access to sources of legal advice. Libraries without access to university legal departments felt that the lack of such a function was an important flaw in the organisation in relation to copyright. Libraries with access to university legal departments felt that these departments tended not to resolve the issues at hand and felt a need for a more specialized legal position dedicated towards copyright.

During some of the interviews the interviewees demonstrated an erroneous understanding of copyright. The result creates additional barriers to open access. Despite the fact that the interviewees were misinformed the result is equally problematic.

These copyright concerns reflected in the interviews show that copyright is perceived as a problem for libraries and that the libraries do not have access to 
adequate training, readily available information, or dedicated legal counsel. The larger universities' concerns become more acute since the larger universities are also those with more researchers and research publications and therefore more likely to come into contact with copyright and licensing questions. In addition to this the larger universities also are more active in open access questions and selfarchiving practices and therefore more likely to come into contact with copyright concerns.

\subsection{Research culture}

The librarians all spoke of the difficulties in changing the culture of the researchers who ignore open access and publish in the traditional journals. Additionally there was little interest among the researchers to attempt to renegotiate with journal publishers in order to preserve their rights or to maintain rights for the university library to self-archive. One interviewee quoted a researcher as saying that open access was unnecessary as all his peers had access to the journals that counted. However, the interviewees also maintained that the researchers are open and sympathetic to the open access idea once it was explained to them.

Therefore the problem with the research culture was therefore not that the researcher could not be convinced but rather the problem was reaching the researchers. It was widely admitted that information via email or paper was not effective since it could be readily ignored. Information meetings were seen as having the best effect but there is also a risk of preaching to the converted and not being able to reach those who are disinterested.

The smaller universities whose researchers either belong to larger research groups or whose researchers are $\mathrm{PhD}$ students with supervisors at other universities are difficult to convince as they tend to follow the norms and recommendations of the external groups or supervisors.

The interviewees all leaned towards a carrot and stick system - the carrot for the researcher being the advantage of additional readers in an open access system. This is the part where the librarians work hard to convince the researchers to adopt open access practices. The stick, almost all libraries think was required, was the need for a strong university leadership to be able to place demands on the researchers to adopt open access practices. An additional stick was seen in the potential of funding bodies to demand that money be connected to open access. Those interviewees who did not feel the need for coercing researchers into open access belonged to the smaller universities with few researchers.

\subsection{Administrative concerns}

The administrative concerns consist partly of the need to convince those involved in adding material to the database or reporting publications that such practices are both relatively easy and well worth carrying out. These tasks are organized and carried out differently at the many departments and faculties at the 
interviewed universities; however, the task falls either on the author, the local administrator, or a librarian. This administrative concern is not considered to be of great importance but it is recognized as being necessary to do something in order to overcome it to ensure widespread self-archiving and reporting of publications.

A more difficult concern related to the administration of self-archiving among librarians is the wrongful use of metadata. In all cases where those entering data were not librarians the high levels of errors among the metadata raised concerns for librarians since this entails additional work for them. However, only in universities with low levels of research publications was it seen as an option to allow librarians to add material. All other universities represented by the interviewees pointed out that this was an impossible task for the librarians to carry out.

A further administrative issue, which occurred in cases where pre-prints were used, was the issue of versions. This problem is one of being able to identify a "correct" version, i.e., ensuring that the archived version is the same as the published version. In most cases the interviewees expressed concerns that researchers did not have an adequate control over different versions of their publications and that self-archiving erroneous or flawed versions would inevitably decrease the value of the archiving system.

The final administrative concern was with dealing with copyright itself. This concern was in two parts: the issue of license changes and the issue of copyrightable content included in the archived publications. The problem with changes to established licenses, either between library and publisher or between author and publisher, was a source of major concern as it was recognized that these changes occurred with little or no warning and the consequences could be great.

The second copyright related concern was that of publications which include copyrightable content. Most interviewees were relatively relaxed in that the archiving required that the author sign a license granting the university use and ensuring that the author had full right to use the publication in this manner. However, certain interviewees expressed concern that they had no way of knowing what the author understood or which content was included in the publication with correctly acquired permission. All universities adopted a "don't ask don't tell" approach in that they adopted a license upon which they relied totally and did not exercise any copyright control.

\subsection{University context}

The differences in size, age, organisation, and culture of the universities all affected the way in which the adoption of open access was proceeding at the different universities. All these factors affect the way in which the university can demand action from its researchers and the way in which the researchers will react towards the demands. 
Generally speaking the newer, smaller universities were better controlled in that the faculties and departments had not developed independently. Older and larger universities tend to be better at resisting centralized control and therefore do not quickly follow the demands of the university to adopt open access.

\subsection{Legal culture}

The legal culture at the university was felt by the interviewees to present a particular set of problems in relation to open access issues. Generally speaking, the universities without access to legal departments to consult felt that this was a major problem, while universities with such departments did not feel that the legal departments understood what the libraries needed.

The latter group realized that the university legal department had many varying administrative tasks and therefore could not be dedicated enough to the questions of open access and copyright to fill the needs of the library.

In addition to this, the role of the legal department is to ensure that any potential liability the university may risk is limited. This position causes the legal department to be negative towards interpreting legal documentation in favour of the libraries needs to adopt open access. This has the effect that the legal departments do not recommend open access and in some cases actively work against such adoption.

In most cases, as described earlier, the universities have a reliance on the copyright licenses entered into between the university and the researcher guaranteeing that the researcher has the right to archive and that all copyrightable material in the publication is there with permission. However in some cases the legal administration of these licenses is carried to such extremes that open access is possible in theory but in practice it is difficult to implement. One such example is the university that demands that such a license be signed by the authors in duplicate and stored physically at the library.

\subsection{Information needs}

Many of the interviewees felt that the resources allocated towards open access were adequate. What was missing was reaching researchers and administrators with more information. It was felt that this information in itself would convince the readers that open access was a worthwhile activity.

These informational needs could be broken down into different types of information. Many interviewees felt that a copyright helpdesk was necessary; others felt that additional documentation was required to educate both librarians and researchers. Many pointed to the lack of courses and seminars that needed to be held in this area. 


\section{Conclusions}

The project is going strong with initial results showing that there is indeed a need for more work in order to help libraries in their transition to adopting effective open access solutions.

The lack of universal access to scientific publications only serves to strengthen the knowledge divides within the knowledge society. In part the lack of access to scientific data has created a wide-ranging growth in the amateur dissemination of data. Projects such as Wikipedia have shown that there is a great universal interest in the dissemination of scientific knowledge. Projects such as Wikipedia also show that if the professional dissemination of scientific data will not adequately provide what people need then alternatives will arise. However, projects based upon amateurs lack the necessary scientific rigor in ensuring the validity of scientific results.

Looking beyond such amateurish attempts we have seen that the economics of scholarly publishing is moving us deeper into the serials crisis and has already forced universities to discontinue subscriptions to necessary scientific publications. Thus, we are building a greater rift between those that can afford scientific data and those who cannot.

Beyond the economics of publishing there also lays an important philosophical approach to open access and this is the fact that the current incumbent system of economic publishing is highly wasteful and drains research resources from the production of research to the dissemination of results. Therefore it is hoped that open access solutions, through methods such as the one based in Lund, will act towards rebalancing the system of scientific research and the dissemination of results.

1. Aside from conducting additional interviews to ensure the validity of the results there are several areas that require work for the future. Some of the areas requiring additional work are: Licensing: All universities use licenses in order to ensure that the researchers' results are archived and can be accessed. These licenses appear in different forms at the different universities. The licenses appear in everything from notices that appear to those entering full texts into the database to the opposite extreme of requiring all authors to physically sign paper documents. No matter what form they appear in the protection afforded by these licenses is questionable and therefore a great deal of work should be carried out in order to streamline and standardize the licensing system in order to strengthen the transition to open access.

2. Documentation: The university libraries require documentation in the form of information brochures and educational documents. These documents are needed to inform librarians, researchers, and administrators. 
3. Licenses: One way to help open access would be to strengthen the position of the author. This can be done by creating licenses and author addendums which the universities can adopt and recommend to their researchers.

4. Education: Among the recurring requests that came up in the interview was the need for further education for librarians. Therefore seminars and lectures should be held to ensure that the level of copyright knowledge among librarians is increased.

5. Policy study: The importance of administrative policies cannot be underestimated and there is a need for a study of the policies and policy documentation available at universities.

6. Helpdesk: Another issue that was raised in the interviews was the need for a copyright and licensing helpdesk. Among the future uses for the project would be to provide this service.

\section{References}

[1] Anderson, B (2004). Open access and institutional repositories. Behavioral \& Social Sciences Librarian 23(1), 97-101.

[2] Argyris, C. Putman, R. \& Smith, D. (1985). Action science: Concepts, methods and skills for research and intervention. Jossey-Bass, San Francisco.

[3] Bailey, C. W. (2005). The role of reference librarians in institutional repositories. Reference Services Review, 33, 259-267.

[4] Baskerville, R. \& Wood-Harper, T. (1996). A critical perspective on action research as a method for information systems research. Journal of Information Technology, 3 (11), 235-246.

[5] Björk, B-C. (2004). Open access to scientific publications - an analysis of the barriers to change? Information Research, 9(2). January.

[6] Dingley, B. (2006). U.S. periodical prices - 2005, U.S. Periodical Price Index 2005, American Library Association.

[7] Eysenbach, G. (2006). Citation advantage of open access articles. PLoS Biology 4(5).

[8] Germonprez, M. \& Mathiassen, L. (2004). The role of conventional research methods in information systems research. In Proceedings of IFIP 8.2 (Kaplan, B. et al Eds.) Kluwer Academic Publishers, Boston.

[9] Harnad, S. et al. (2004). The access/impact problem and the green and gold roads to open access. Serials Review 30(4), 310-314.

[10] Harris, M. (2005). Institutional repositories: is the open access door half open or half shut? Learned Publishing 18(2), 85-90.

[11] Hedlund, T., Gustafsson, T. \& Björk, B-C. (2004). The open access scientific journal: an empirical study. Learned Publishing 17(3), 199-209.

[12] Holwell, S. (2004). Themes, iteration, and recoverability in action research. In Proceedings of IFIP 8.2 (Kaplan, B. et al Ed.) Kluwer Academic Publishers, Boston.

[13] Kaufman P. (1995). Why we must subscribe to fewer journals. Information Issues. University of Tennessee, Knoxville Libraries.

[14] Klang, M. (2006). Disruptive technology: Effects of technology regulation on democracy, Doctoral Dissertation, University of Göteborg. Defended publicly 2nd October 2006.

[15] Kock N. (1997). Myths in organizational action research: Reflections on a study of computer-supported process redesign groups. Organizations and Society 4 (9) 65-91.

[16] Lincoln, Y. \& Guba E. (1985). Naturalistic enquiry. Sage Publications, London. 


\section{Social Dimensions of ICT Policy}

[17] Peters, J. (1995). Hard choices for the libraries' collections. Information Issues. University of Tennessee, Knoxville Libraries.

[18] Rapoport, R. (1970). Three dimensions in action research. Human Relations 23 (4), 499-513.

[19] Susman, G. \& Evered, R. (1978). An assessment if the scientific merits of action Research. Administrative Science Quarterly (23), 582-603.

[20] Tenopir, C. (2004). Online scholarly journals: How many? Library Journal 129 (2), 32.

[21] Van Orsdel, L. C. \& Born, K. (2005). Choosing sides: Periodical price survey 2005. Library Journal, April.

[22] Whyte, W. (1991). Participatory action research. Sage Publications, Newbury Park, CA.

[23] Willinsky, J. (2006). The access principle: The case for open access to research and scholarship. MIT Press, Cambridge Mass. 